\title{
3D VIRTUAL RECONSTRUCTION OF AN URBAN HISTORICAL SPACE: A CONSIDERATION ON THE METHOD
}

\author{
M. Galizia ${ }^{a}$, C. Santagati ${ }^{\text {a }}$ \\ ${ }^{a}$ Dept. of Architecture, Engineering Faculty, 95125 Catania, Italy \\ mgalizia@dau.unict.it \\ cettina.santagati@dau.unict.it
}

KEY WORDS: laser scanning, 3D modeling, knowledge, urban space

\begin{abstract}
:
Urban historical spaces are often characterized by a variety of shapes, geometries, volumes, materials. Their virtual reconstruction requires a critical approach in terms of acquired data's density, timing optimization, final product's quality and slimness.

The research team has focused its attention on the study on Francesco Neglia square (previously named Saint Thomas square) in Enna. This square is an urban space fronted by architectures which present historical and stylistic differences. For example you can find the Saint Thomas'church belfry (in aragounese-catalan stile dated XIV century) and the porch, the Anime Sante baroque's church (XVII century), Saint Mary of the Grace's nunnery (XVIII century) and as well as some civil buildings of minor importance built in the mid twentieth century.

The research has compared two different modeling tools approaches: the first one is based on the construction of triangulated surfaces which are segmented and simplified; the second one is based on the detection of surfaces geometrical features, the extraction of the more significant profiles by using a software dedicated to the elaboration of cloud points and the subsequent mathematical reconstruction by using a $3 \mathrm{~d}$ modelling software. The following step was aimed to process the virtual reconstruction of urban scene by assembling the single optimized models.

This work highlighted the importance of the image of the operator and of its cultural contribution, essential to recognize geometries which generates surfaces in order to create high quality semantic models.
\end{abstract}

\section{INTRODUCTION}

This study is the result of research work carried out at the "Luigi Andreozzi" Laboratory of Architectural Photogrammetry and Survey in order to understand and document the local architectural and environmental heritage.

In this area of study, the research team has improved the operational methodologies of the survey as well as of the representation of the historicised urban spaces for their virtual reconstruction.

The historical piazzas are often characterised by stratifications which have shaped the urban environment through a variety of shapes, geometries, volumes, and material over time. Their virtual reconstruction, thus, requires a critical/analytical approach in terms of amount of acquired data, optimisation of time, and quality as well as manageability of the final product.

From this point of view, the city of Enna still bears evidence of the medieval urban fabric thanks to its historical events and its environmental characteristics thus offering ideas for research. In the irregular road system of Enna, Piazza Paolo Francesco Neglia (ex Piazza San Tommaso) - an urban enclosed area upon which historically and stylistically different architectural structures rise - stands out. Among these structures, we can find the Catalan-Aragonese style bell tower (17th c.), which leans against the porch and the church of San Tommaso, the baroque church of the Anime Sante (17th c.) as well as the Convent of Santa Maria della Grazia (18th c.); the piazza ends with some less important houses which were built in the mid-twentieth century.

Experiments conducted on the rich architecture of this urban enclosed area (the bell tower and the porch of the church of San
Tommaso, the church of the Anime Sante) have compared two different methodological approaches which are aimed at the modelling of data obtained from a 3D laser scanner: the first approach is based on the construction of segmented and simplified triangulated surfaces; the second approach is based on the identification of the geometric characteristics of the surfaces, the extraction of the most significant profiles, and the mathematical reconstruction of the surfaces.

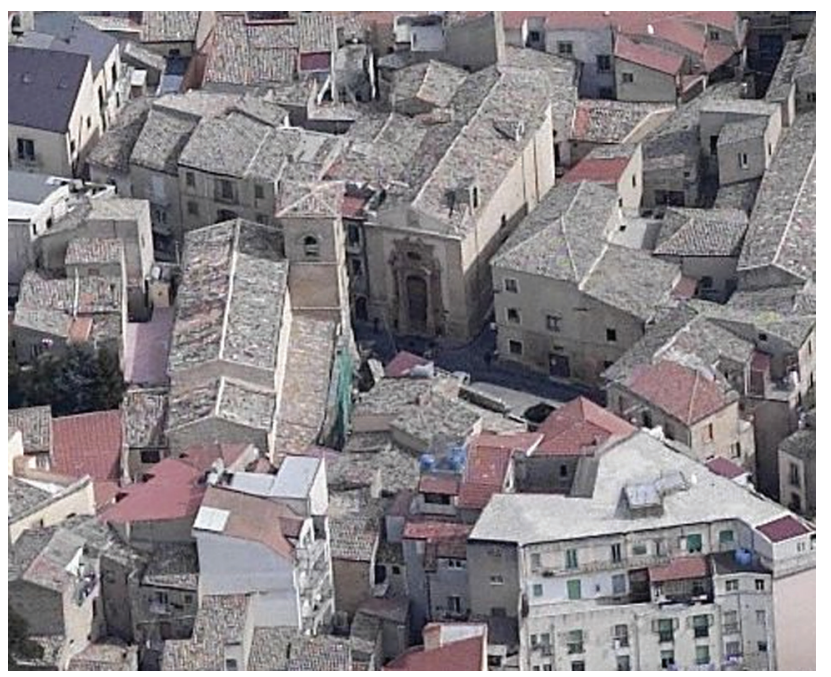

Figure 1. Piazza Paolo Francesco Neglia urban historical space 
The two procedures have been applied to the single pieces of architectural handiwork, regardless of their geometric/formal complexity, in order to verify the effectiveness of their results in terms of time of processing, quality and consistency of the files and perfection of the produced model. The processing of the virtual reconstruction of the urban setting has, then, been carried out by assembling the single optimised models.

The three main phases of the experimentation were:

- planning a survey of the architecture through a 3D laser scanner, acquisition of data and subsequent georeferencing.

- processing data according to two identified methodological approaches;

- geometric analysis and data comparison.

\section{SURVEY AND DATA PROCESSING}

The data acquisition project was carried out by taking into consideration the distinctive features of the site and the characteristics of the equipment employed.

In particular, the selection of the standpoints was strongly influenced by the heavy pedestrian and road traffic which characterises the area, and by the vehicles parked along the perimeter of the piazza which made the acquisition of the ground connections of the buildings difficult. Indeed, in order to obtain a full coverage of the basin of the piazza, special attention was paid both to the possible grey areas generated by the bodies found between the scanner and the object of interest, and to the necessity of having good superimpositions between the different scanned images, so as to be able to perform the subsequent union of the clouds through homologous points.

The Leica Geosystem HDS 3000 TOF 3D scanner laser was used, since it is particularly suitable for the distance survey of large buildings with a precision range $(+/-4 \mathrm{~mm}$, and an angle precision of 60 microrad) and scanning field equivalent to $360^{\circ}$ horizontally and to $135^{\circ}$ vertically.

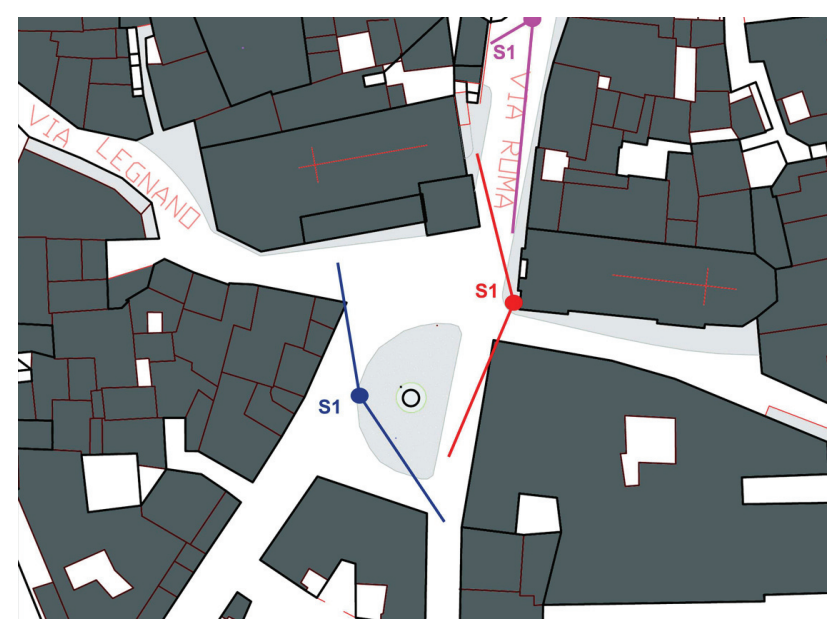

Figure 2. Data acquisition project

Three standpoints were selected with a step-scan ranging from 5 to $15 \mathrm{~mm}$ so as to achieve a good compromise between the density of the obtained points, the level of detail and the optimisation of the time of data acquisition in situ.

Once metric data were acquired, the following step was to put together the whole model through the assembling of the scanned images which were then georeferred to just one system of reference.

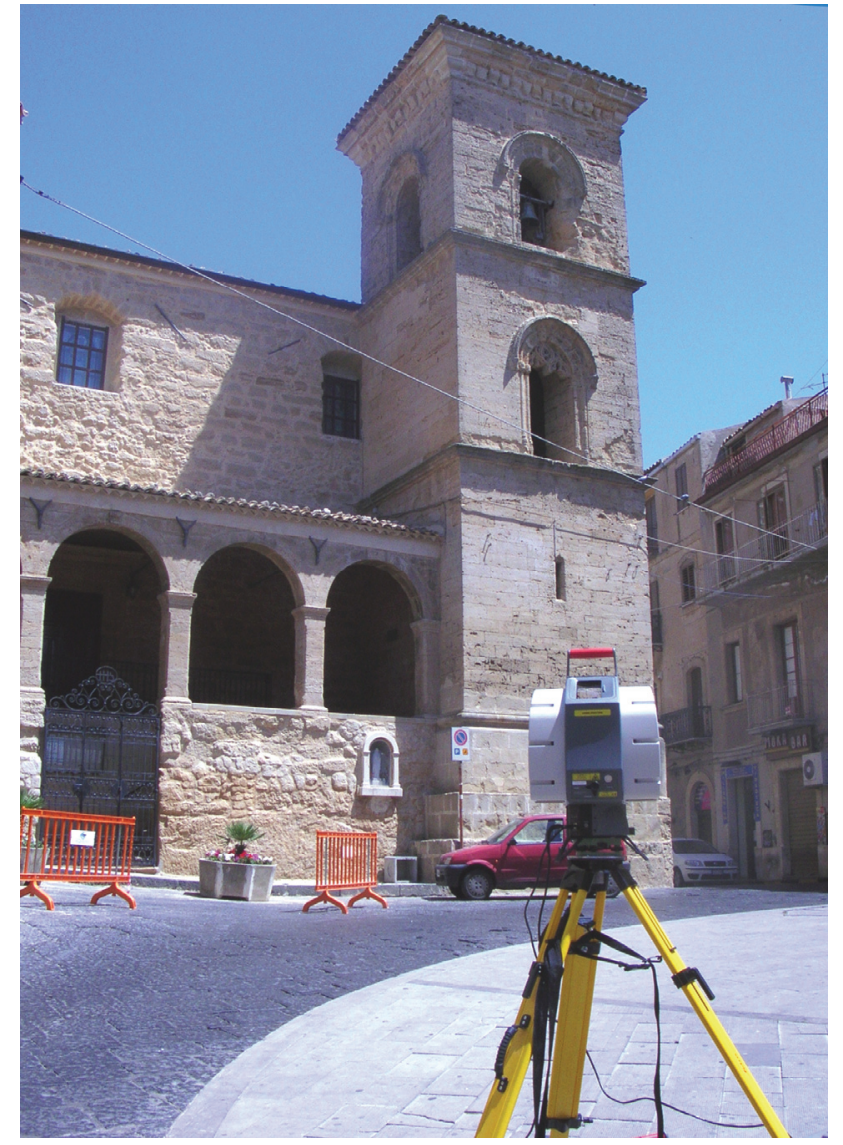

Figure 3. In situ survey

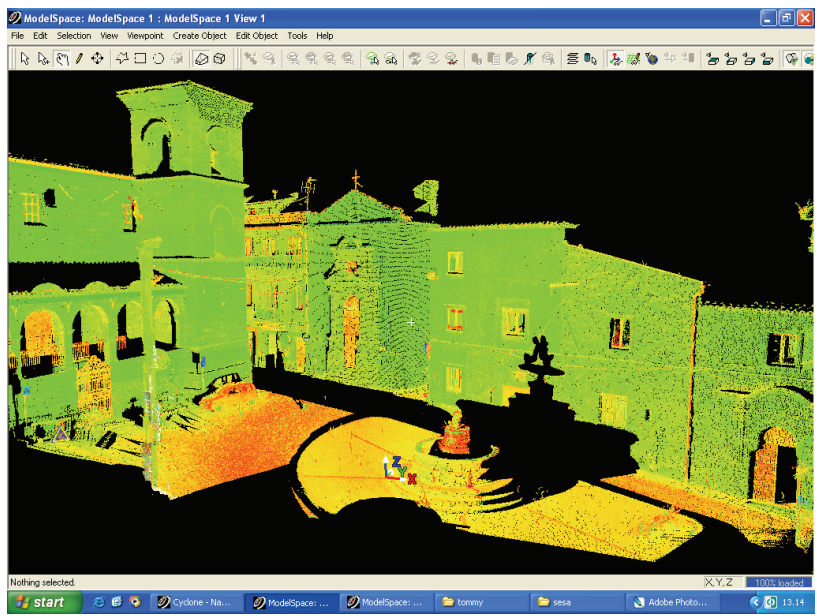

Figure 4. View of Francesco Neglia square's point cloud

After that, operations continued manually through the identification of homologous points on pairs of scanned images, by linking together each scanned image to the previous one using the polygonation method.

Calculation parameters (subsample percenting, number of interactions, max search distance) were optimised in order to minimise alignment error between scanned images which on average was about $2 / 3 \mathrm{~mm}$. 
The complete three-dimensional model of Piazza Neglia consists of the combination of the three scanned images to the amount of 33 million points.

Elements which created disturbance such as passers-by, moving and parked vehicles, as well as black spots generated by a bad return signal of the laser ray, were then eliminated from the global model.

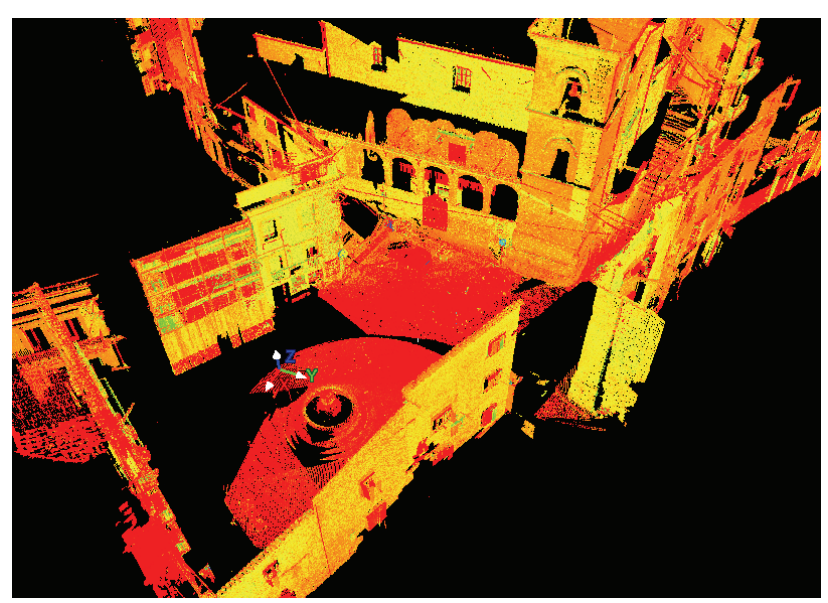

Figure 5. View of the assembled Francesco Neglia square's point cloud model

\section{DATA PROCESSING: TWO METHODOLOGIES COMPARED}

\subsection{Concept}

The cloud of points obtained from the 3D laser scanner represents a numerical model of reality upon which further studies and interpretations can be carried out according to the set goals.

The great amount of data to be processed is well suited to experimentation and comparison between different approaches to modelling according to the formal and stylistic qualities of the analysed object, to the purposes of the survey as well as of the representation, and, thus, to the level of detail.

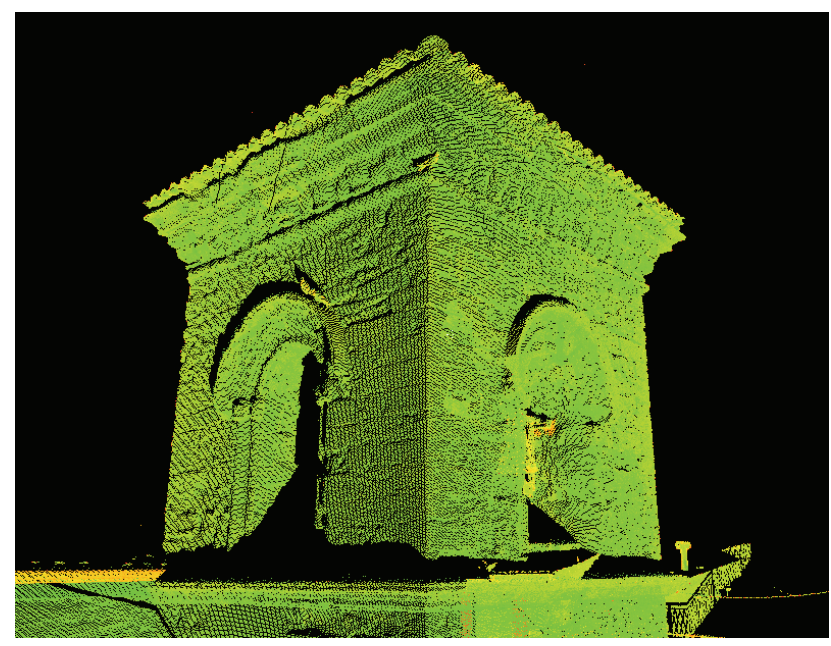

Figure 6. Detailed view of Saint Thomas' belfry
What is the best direction to take? What are the parameters underlying the choice of the project of the descriptive model to be created? Which data should be selected?

The objective is the comparison between two operational methodologies which aim at the virtual reconstruction of Piazza Neglia through a model which is able to document the urban space both qualitatively and quantitatively. This comparison takes into account the following important aspects: manageability of the file, processing time, final quality of the model.

For the reconstruction of the surfaces of the architecture, two different methods were adopted and they were based on: polygonal meshes generated by the topological optimisation of acquired data; mathematical surfaces generated by geometric algorithms underlying the shape which was built.

Once the parameters had been fixed, they were tested on each architectural unit which, due to its geometric and formal characteristics, represented a specific case study to be investigated individually as well as according to the mutual relationship with the environment in which it is placed.

\subsection{Triangulated modelling}

Modelling for triangulated surfaces allows us a greater control of the "approximation" of reality in virtual models.

Basically, through software for the creation of clouds of points, a selection of data is made thanks to an "intelligent" choice of points, which is bound to parameters the operator himself has indicated according to the qualitative aspects of the architecture. In this case study, the architecture of Piazza Neglia presents such characteristics that segmentation into homogeneous portions was necessary in order to create representations closely connected to the physical qualities of the handiwork according to weight and definition.

For this purpose, the flat surfaces of the battlements of the church, of the tower, and of the porch were dealt with differently from the architectural elements, which present a more complex geometry: the double mullioned window, the single mullioned window, the string courses, the brick cornice of the tower, and the porch of the church.

The single clouds of points, which were obtained by "cropping" the original cloud, underwent a "data-filtering" process during which some parameters connected to the characteristics of the real surfaces were processed.

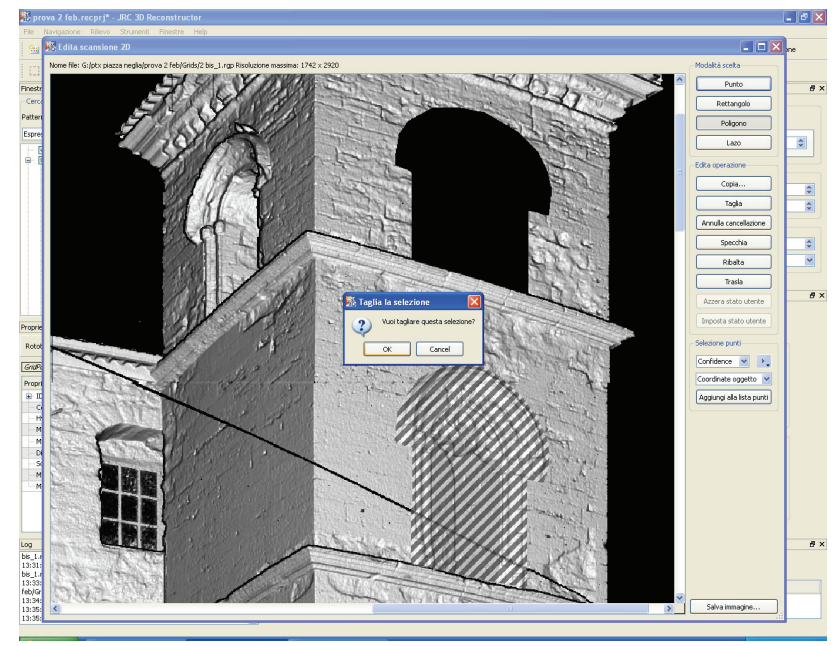

Figure 7. Segmentation into homogeneous portions by "cropping" the original cloud 
A mesh was created on every single pre-processed cloud by changing each time the values of accuracy, max triangle size, min triangle aspect ratio, and angular discontinuity according to the geometric complexity.

Indeed, the triangular meshes can often be too complex to be managed by computer, and, thus, it is essential, when it is possible, to simplify them. This is what was done to those wall surfaces for which assimilation to one plan made it possible to use values which could produce a larger mesh, yet retaining the definition of the consistency of stone.
A different approach was used for the architectural details since the plastic mouldings and the sculptural elements required the creation of a dense triangular mesh. During this phase, the coverage of existing depth discontinuities (holes) which are caused by the shadows of projecting parts was avoided by matching points at a distance. For this reason, it was necessary to eliminate triangles with too long sides as well as with particularly acute angles.
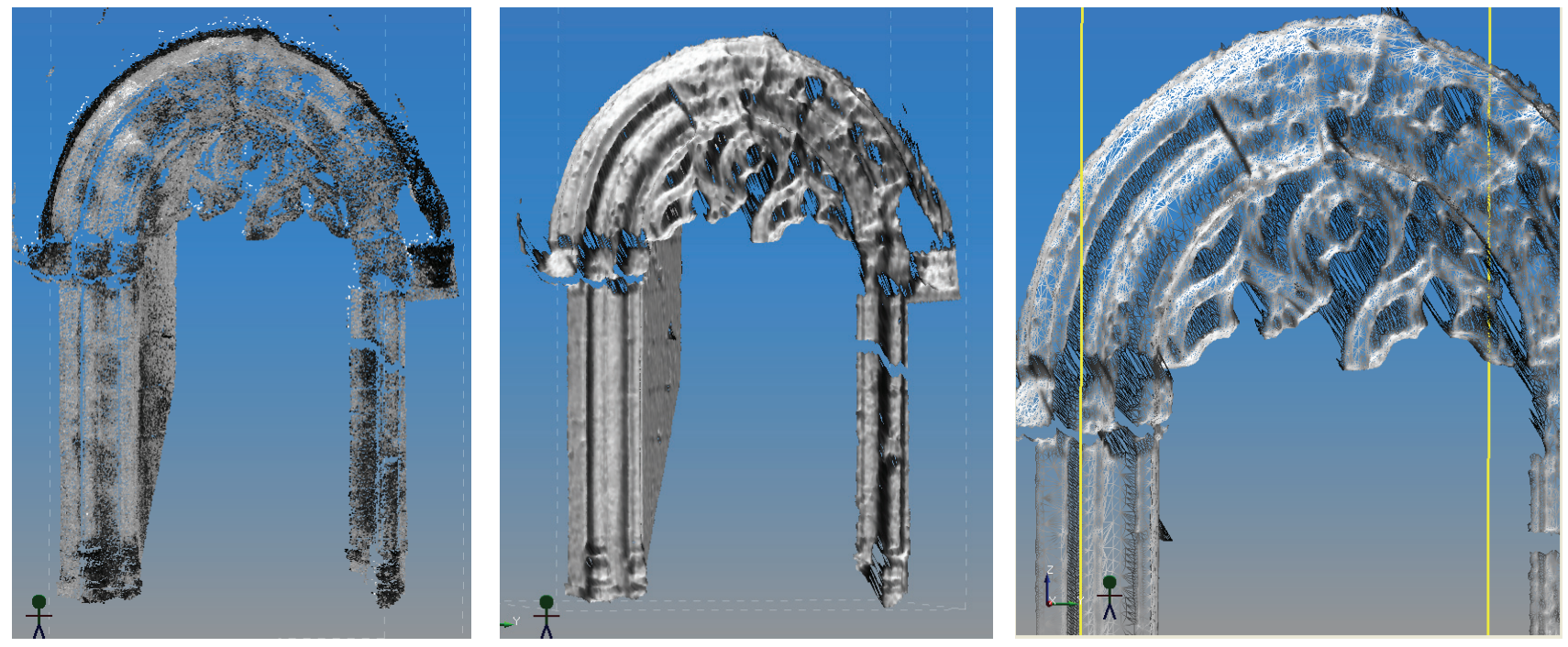

Figure 8. From the cloud point to the triangulated model of Saint Thomas' bell tower duble mullioned window

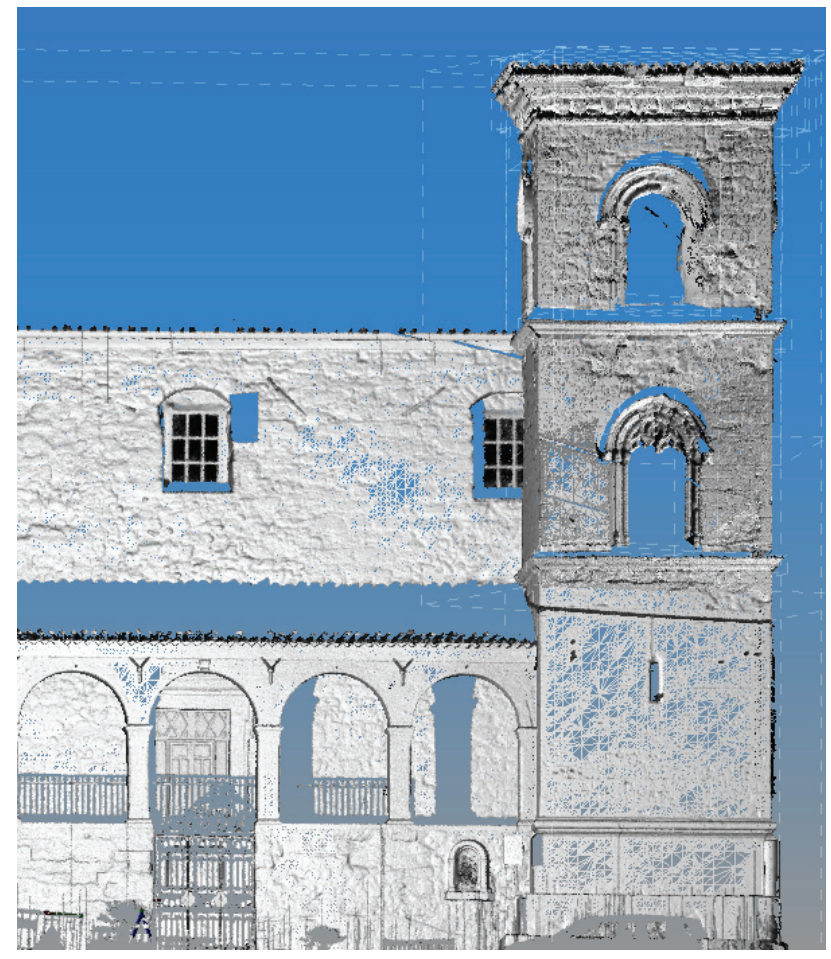

Figure 9. Porch and bell tower poligonal model
In this way the model, though it seems to be lacking information, is very faithful to its numerical model, and, thus, to the observed reality, without any approximation.

The creation of the single triangulated models converted the cloud of points into a polygonal surface, which produces a visually and perceptively more effective representation in which deterioration, instability, and missing parts of the architectural handicraft can be easily observed.

The model, which was obtained, in a relatively short time, by assembling single parts, is a faithful representation of reality from which it is possible to get geometrico-dimensional information.

In addition, the 3D data of a metric nature were integrated with 2D information acquired through the camera. The mapping of the photographic texture of the triangulated surface helped to create a highly detailed representation of the most important architectural elements, without making the original file too heavy and thus improving its manageability. 


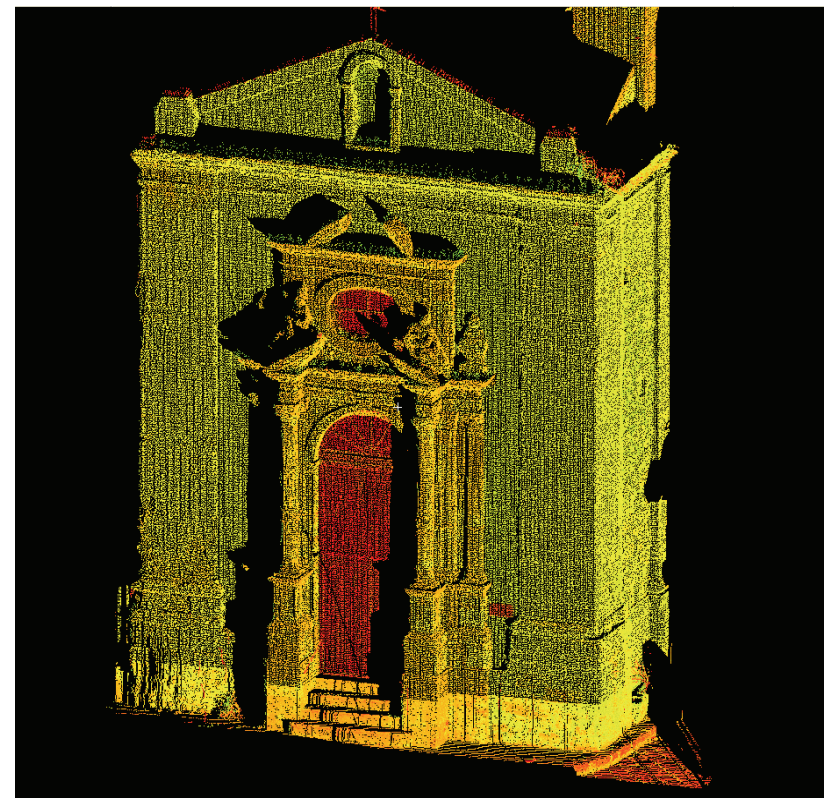

Figure 10. Detailed view of Anime Sante's church

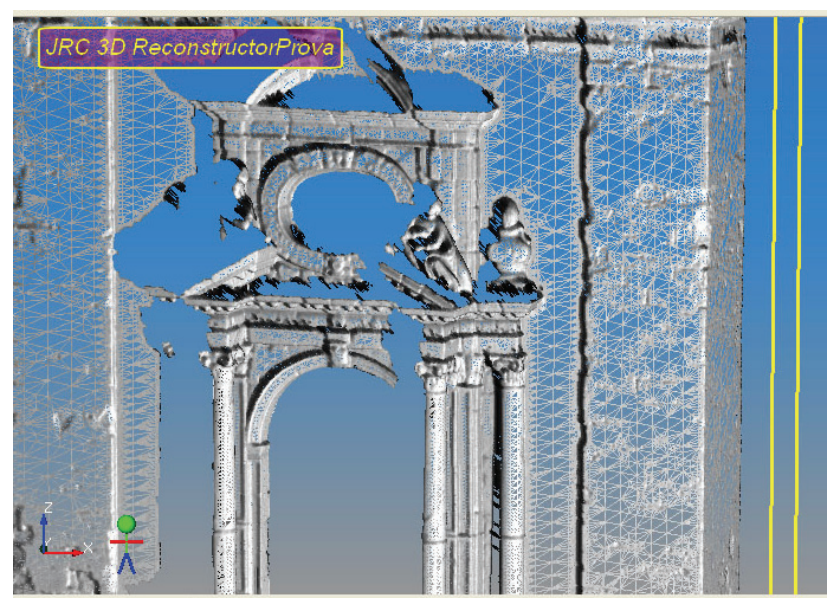

Figure 11. Anime Sante's church poligonal model

\subsection{Geometric modelling}

The passage from the numerical model formed by the cloud of points to the final geometric model made necessary the selection and interpretation of data, which aimed at their representation. Due to the great amount of information provided by the cloud of points, the operator has to make some choices, as well as fixing the level of approximation between reality and its geometric abstraction. The cultural responsibility of the operator is even more crucial when there are highly deteriorated elements with detachments and missing parts.

In this case, preliminary understanding of the constitutive geometry of each single architectural detail helps to make a correct discretization as well as identifying the most significant profile sections which are useful to describe their surface effectively.

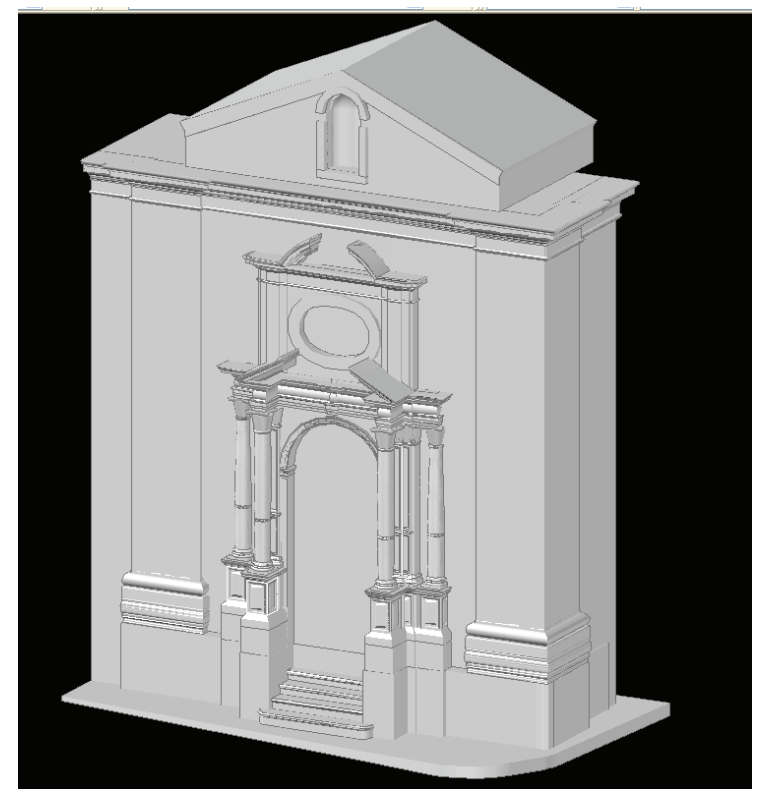

Figure 12. Rendering of Anime Sante's church geometric model

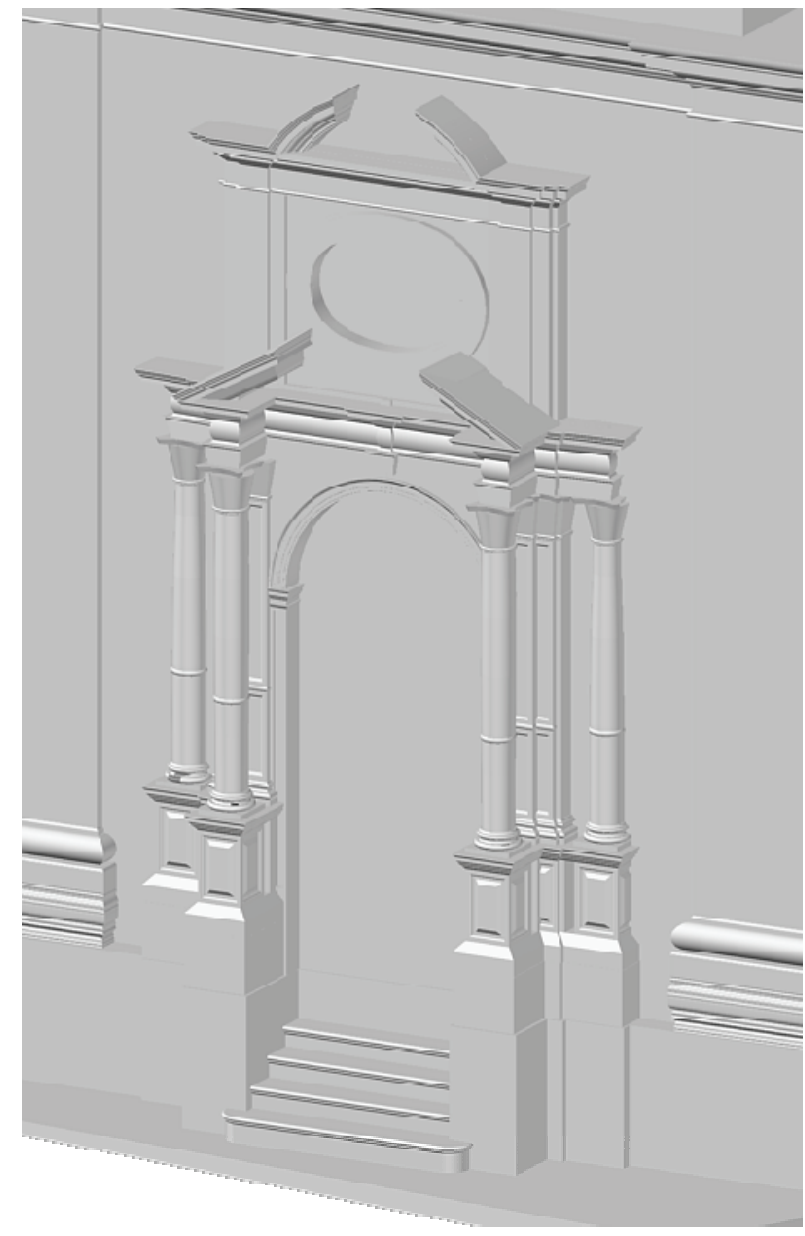

Figure 13. Detailed view of the baroque portal of Anime Sante's church geometric model 
Therefore the operator, thanks to his experience, skills and the ability to interpret the geometric "rule" underlying the architecture, plays the most important role in this process. Indeed, the models he creates have a high semantic content in which each element is described exhaustively by the geometric shape which constitutes its structure.

Experimentation on identified case studies - architecture characterised by geometric elements of a different complexity has offered the opportunity to expand and verify the said observations.

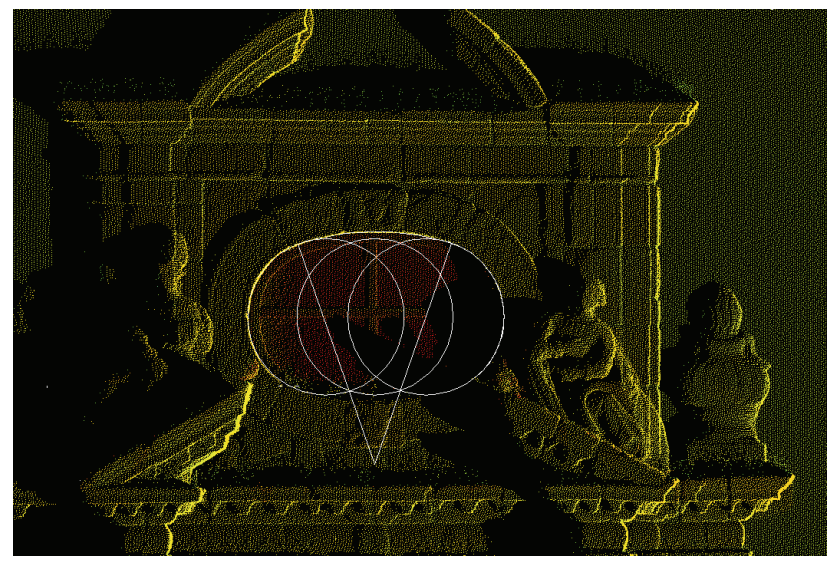

Figure 14. Geometric construction applied to the oval-shaped window

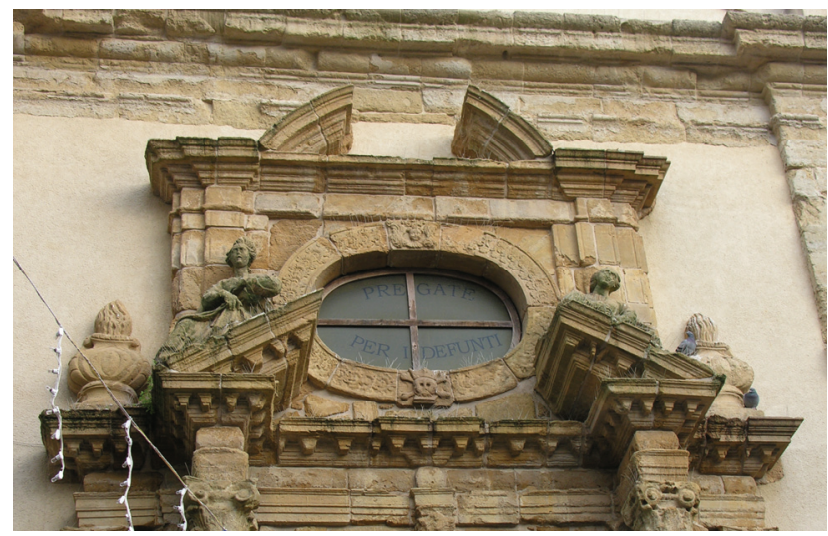

Figure 15. Detailed image of the portal entabled

In particular, $\mathrm{CAD}$ technology was used by operating directly on the cloud of points. In this way a direct verification of the performed operations was obtained, and, through appropriate slices on the cloud of points, coherence between the geometry of the model and the observed reality was verified.

During the modelling of the façade of the church of the Anime Sante, great attention was paid to the rich geometry of the baroque portal, thus choosing to make a discretization which would retain the most accurate information about architectural details. From the operational point of view, the constitutive elements of the architectural order (pedestal, column, entablature) and the relevant generating geometries were identified. Through appropriate slices on the cloud of points, the significant profiles of the various elements and of the detailed mouldings were redesigned. The following step led to the reconstruction of the surfaces through adequate mathematical algorithms.

The same procedure was applied to the bell tower of San Tommaso. In this case, the highly square-shaped geometry of this architecture which is divided into three levels by string courses made it necessary to work by single portions, thus optimising time. Also in this case attention was especially focused on the decorative elements such as the double mullioned window on the second floor and the single mullioned windows on the third floor.

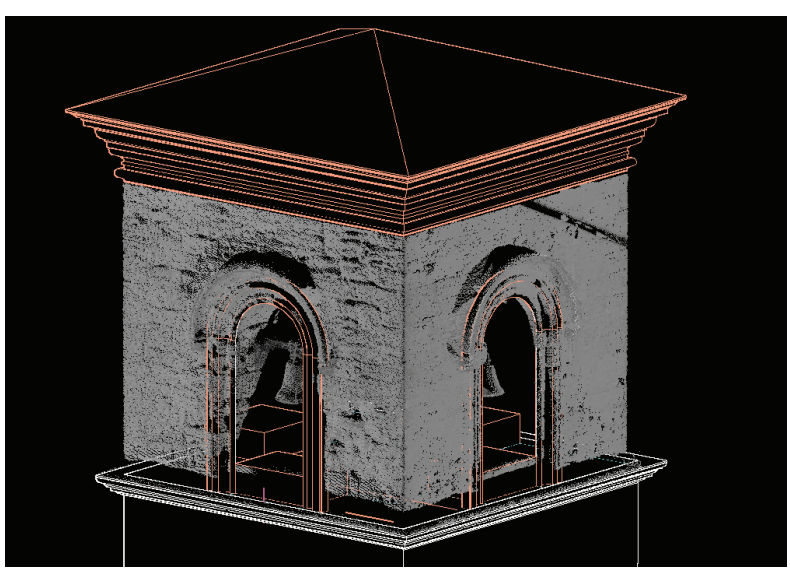

Figure 16. From the cloud point to the geometric model of Saint Thomas's belfry third level

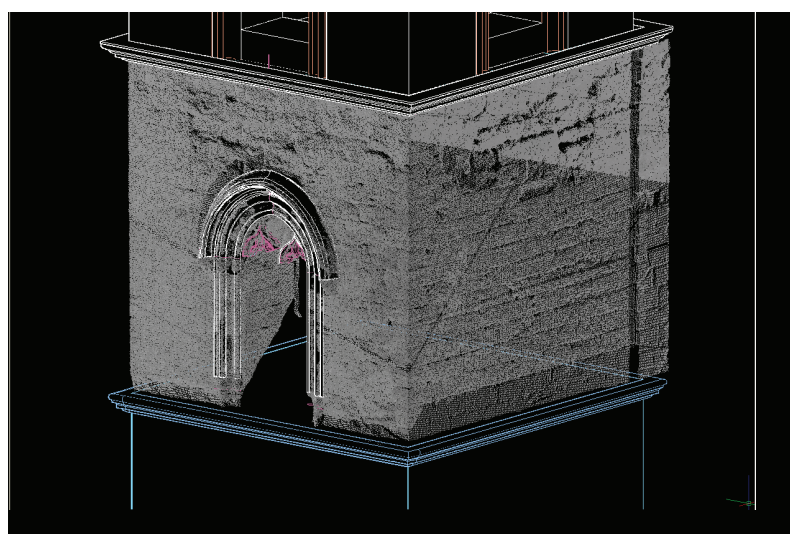

Figure 17. From the cloud point to the geometric model of Saint Thomas's belfry second level

Finally, the modelling of the portal of the church measured by the pillars which support lancet arches involved a remarkable geometric abstraction of the base, which was particularly damaged. The remaining surfaces were then again discretized so that all architectural information details were preserved.

The resulting model of urban environment, thus, describes the observed reality geometrically, by representing it through a selection as well as a schematisation of the architectural elements which form it.

\subsection{Considerations}

The three-dimensional digital models, which have been obtained by applying the two different procedures, document the urban space, each one analysed with a different level of indepth examination.

The advantages and limits presented by such models are closely connected to the procedures which generated them. 


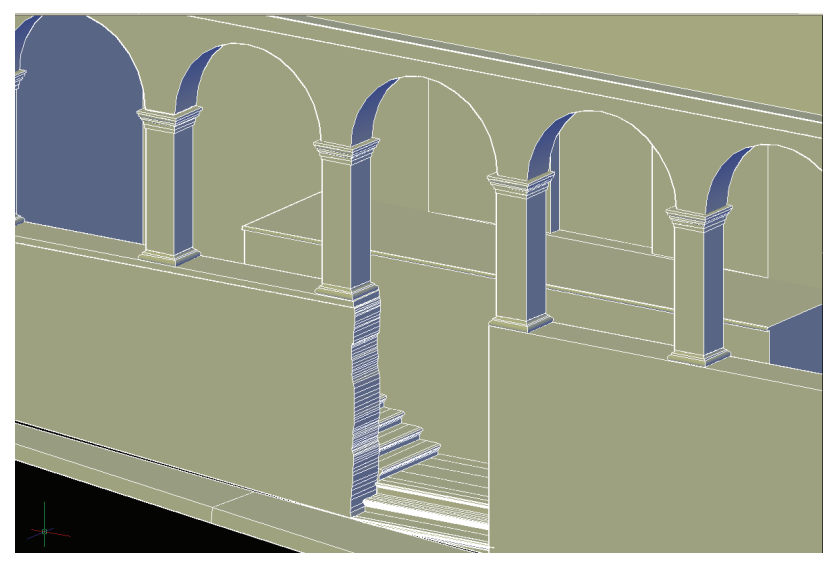

Figure 18. View of the porch geometric model

Indeed, speed in terms of time and simplicity of optimisation of the parameters necessary to obtain a polygonal model of a good quality contrast with the lack of information about the geometric genesis of the documented surfaces.

Moreover, the description of the surfaces through triangular meshes rather than mathematical algorithms involves an excess weight of the file. On the other hand, the triangulated surface retains countless information on the state of conservation of the heritage material useful for a comprehensive documentation.

On the contrary, a greater use of time for the processing, which is necessary for the construction of a good quality geometric model with a level of architectural detail, is compensated for by the semantic quality of the model itself, in which every element is linked to its geometric genesis. In addition, the description of the surfaces through mathematical algorithms results in a less heavy file.

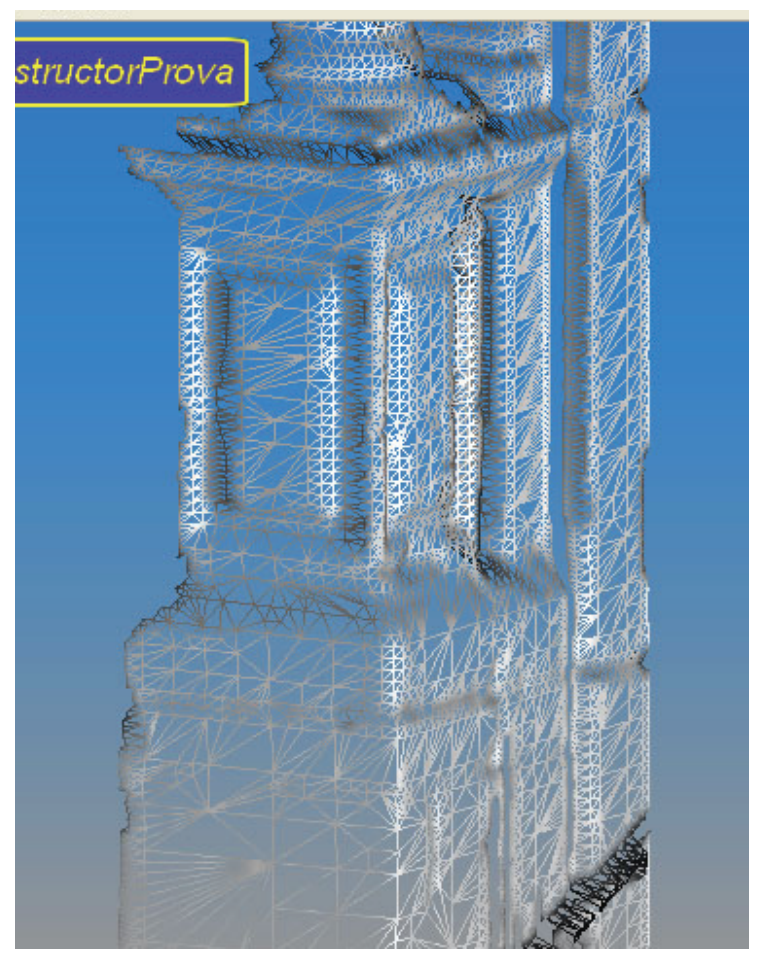

Figure 19. Pedestal polygonal model
Finally, the geometric model is an abstraction of reality, a discretization into parts which often simplifies the complexity of the components of the analysed object.

In relation to the considerations made, it is possible to state that the two models thus obtained, each one with its own limits and advantages, can be considered as complementary in the process of understanding cultural heritage.

\section{CONCLUSIONS}

This work is a methodological reflection on the way to deal with a process of understanding a historical urban space. Indeed, the architecture which characterises the analysed environment, in spite of the deterioration of some parts, succeeds in preserving highly recognizable features. This has made possible the creation of a three-dimensional model faithful to the different architecture in its single constitutive elements, by proceeding through the two different methodological procedures.

Indeed, the obtained models are satisfactory for the understanding and documentation of this highly historicised urban enclosed area.

To this day, then, it is possible to emphasise the importance of the role of the operator who has to take part in the delicate passage from the cloud of points to the virtual model, by selecting and processing the necessary data thanks to his deep knowledge of the architectural shapes.

The results achieved through the comparison of these two methodological procedures will be, in the future, an area of investigation to be applied to the field of archaeology. Indeed, consistency in a state of ruin often involves a greater responsibility on the part of the data collector who is then given the difficult responsibility of the choice of the right way to work, so that what is reproduced by the model could be as faithful as possible to reality, while seeking a geometric abstraction.

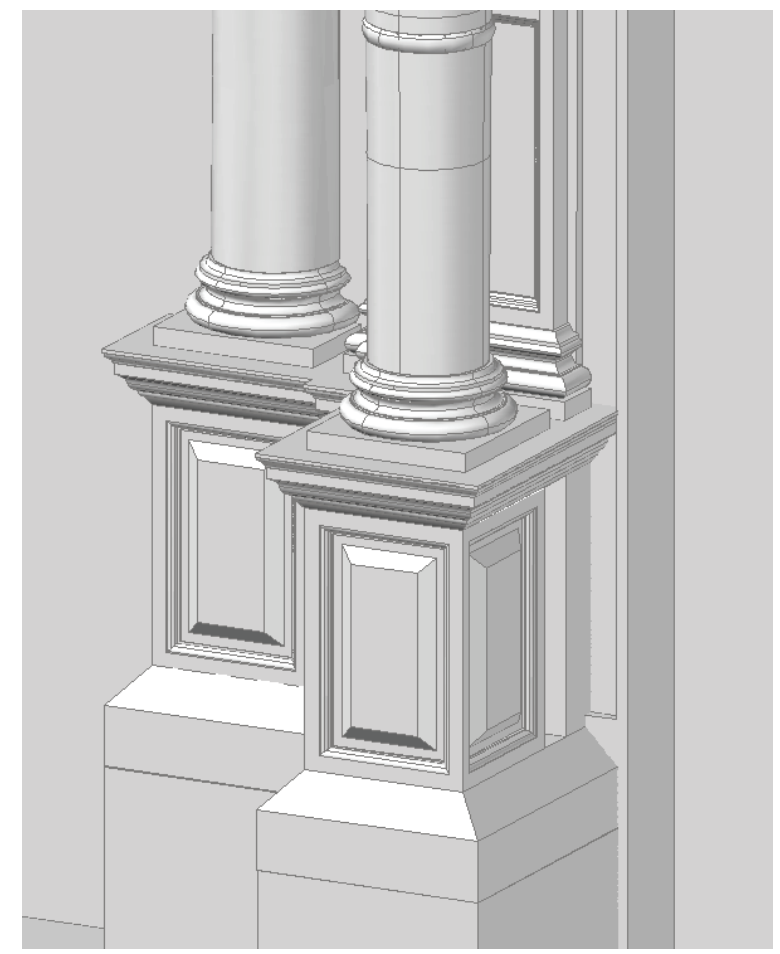

Figure 20. Pedestal geometric model 


\section{REFERENCES}

Apollonio F.I., Gaiani M., Corsi C., 2010. A semantic and parametric method for 3D models used in 3D cognitiveinformation system. In: Future cities. 28th eCAADe 2010 Conference, ZURICH, ECAADE - ETH Zurich, pp. 863 - 872.

Bianchini C., 2009. Towards a systematic theoretical approach for archeology survey and modelling. In: Proceedings of CAA 2009 Conference "Making History Interactive", Williamsburg.

Blaise J.-Y., Dudek I., De Luca L., 2009. Handling mouldings in historic architecture: a matter of geometry, a matter of knowledge? In: Proceedings of CIE39 - International conférence on Computers and Industrial Enginnering, Troyes.

Symposium International cooperation to save the world's cultural heritage, pp. 289-294, Torino.

Guerra F., Balletti C., Vernier P., 2008. La memoria digitale della forma geometrica: scanner $3 \mathrm{~d}$ e fotogrammetria digitale per la realizzazione di modelli virtuali, per la riproduzione, per la conservazione e per la riattualizzazione di oggetti. In: Atti della $12^{\wedge}$ conferenza nazionale ASITA, L’Aquila.

Guerra F., Adami A., Vernier P., Tsiukas V., 2008. Il laser scanner per la verifica di ipotesi geometriche: applicazione ad un monastero musulmano a Xanthi. In: Atti della $12^{\wedge}$ conferenza nazionale ASITA, L'Aquila.

Remondino, F., Girardi, S., Gonzo, L., Rizzi, A., 2008. Multiresolution modeling of complex and detailed Cultural Heritage. In: Proceedings of 9th Int. Symposium on Virtual Reality, Archaeology and Cultural Heritage (VAST 2008), pp. 1-8, Braga, Portugal.

Santagati C., 2005. 3D Laser Scanner aimed to architectural heritage survey: from the point's cloud to the geometrical genesis determination. In: ISPRS International Archives of Photogrammetry, Remote Sensing and Spatial and Information Sciences, VOLUME XXXVI, PART 5/W17 Proceedings of the ISPRS Working Group V/4 Workshop 3D-ARCH 2005: "Virtual Reconstruction and Visualization of Complex Architectures", Mestre-Venice.

Santagati, C., 2007. Il reale e la sua rappresentazione attraverso l'informatica, Il Lunario, Enna.

Galizia M., Andreozzi L., 2005. The survey of decorative elements with laser scanner. In: CIPA 2005 XX International 